https://doi.org/10.15407/ujpe64.7.624

T. ROSTOMYAN

for the MUSE Collaboration

Rutgers, The New Jersey State University

(Piscataway, New Jersey 08855, USA; e-mail: Tigran.Rostomyan@psi.ch)

\title{
STATUS OF THE MUSE EXPERIMENT
}

The $5.6 \sigma$ difference in proton radii measured with $\mu p$ atoms and with ep atoms and scattering remains an unexplained puzzle. MUSE will measure the $\mu p$ and ep elastic scatterings in the same experiment at the same time. The experiment determines cross-sections, two-photon effects, form-factors, and radii and allows $\mu p$ and ep to be compared with reduced systematic uncertainties. These data should provide the best test of the lepton universality in a scattering experiment to date, about an order of magnitude improvement over previous tests, a $\% \sigma$ radius determination, and improved two-photon measurements.

Keywords: proton radius puzzle, MUSE, elastic scattering, muon beam, TPE.

\section{Introduction}

In 2010, a Paul Scherrer Institute (PSI) experiment [1] reported that the proton charge radius determined from muonic hydrogen level transitions is $0.84184 \pm 0.00067 \mathrm{fm}$, about $5 \sigma$ off from the nearly order-of-magnitude less precise non-muonic measurements. This "proton radius puzzle" was confirmed in 2013 by a second muonic hydrogen measurement [2] of $0.84087 \pm 0.00039 \mathrm{fm}$. Subsequent $e p$ scattering results of $0.879 \pm 0.008 \mathrm{fm} \mathrm{[3]} \mathrm{and}$ $0.875 \pm 0.010 \mathrm{fm}[4]$ confirmed the puzzle. The situation has been discussed in review papers [5], in dedicated workshops [6-8], and in many talks. It is generally agreed that new data are needed to resolve the puzzle.

The MUon Scattering Experiment (MUSE) collaboration was formed in 2012 to uniquely attempt to resolve the "Proton radius puzzle" by simultaneously measuring the $\mu p$ and $e p$ elastic scattering cross-sections at the sub-percent level. MUSE alternates between positive vs. negative charged beams all previous measurements are with negative leptons. Thus, MUSE directly compares $\mu p$ to ep crosssections and radii, provides the first significant $\mu p$ scattering radius, and measures two-photon exchange effects (TPE) at the sub-percent level, rather than using the calculated corrections.

(c) T. ROSTOMYAN, 2019

\section{PSI Beam Line} and $\pi M 1$ Experimental Area

The PSI High Intensity Proton Accelerator (HIPA) primary protons strike the $\mathrm{M}$ production target and generate secondary $e^{ \pm}, \mu^{ \pm}$, and $\pi^{ \pm}$beams that are transported through the PiM1 channel to MUSE. Particle species are identified by timing relative to beam RF (Figure 1). The beam composition delivered to $\pi M 1$ is shown in the Table.

Three different beam momenta are chosen to optimize both $e$ and $\mu$ fluxes and RF time separation and provide redundant cross-sections as a check of the systematics. Figure 1 shows that the different particle species are 3-6 ns apart, much larger than the intrinsic timing width of $\approx 300 \mathrm{ps}$.

\section{MUSE Detector Setup}

MUSE is implemented as a set of detectors and cryotargets mounted on a moveable platform, so that

The measured $\pi M 1$ beam composition

\begin{tabular}{|c|c|c|r|r|}
\hline$P, \mathrm{MeV} / \mathrm{c}$ & Polarity & $e, \%$ & $\mu, \%$ & $\pi, \%$ \\
\hline 115 & + & 96.7 & 2.1 & 0.9 \\
153 & + & 63.0 & 12.0 & 25.0 \\
210 & + & 12.1 & 8.0 & 79.9 \\
115 & - & 98.5 & 0.9 & 0.6 \\
153 & - & 89.9 & 3.2 & 6.8 \\
210 & - & 47.0 & 4.0 & 49.0 \\
\hline
\end{tabular}

ISSN 2071-0194. Ukr. J. Phys. 2019. Vol. 64, No. 7 
the PiM1 area can be shared. Figure 2 shows the experiment as represented in a Geant4 [9] simulation. The beam strikes the Beam Hodoscope $(\mathrm{BH})$ detector and three Gas Electron Multiplier (GEM) chambers, passes through a central hole in the annular Beam Veto detector, enters the cryotarget vacuum chamber, strikes one of the targets (Liquid Hydrogen, Carbon, etc.), and then exits the vacuum chamber. Unscattered particles go through the Beam Monitor (BM), while scattered particles are detected by two symmetric spectrometers, each with two Straw-Tube Trackers (STT-s) and two planes of Scattered Particle Scintillators (SPS). The BH identifies particles through the RF timing; triggering depends on the particle type. Reaction identification (scattering vs. decays in flight) uses GEM and STT tracking along with the time of flight (TOF) from the BH to SPS. The BM can also be used to suppress Moeller events. More details are below.

\subsection{Beam hodoscope}

Purpose:

The $\mathrm{BH}$ provides timing information that, along with the RF signal, determines beam particle species for triggering and analysis. TOF from the $\mathrm{BH}$ to the SPS determines the reaction type, in particular, separates the muon decay from the muon scattering. TOF from the $\mathrm{BH}$ to the $\mathrm{BM}$ identifies backgrounds and determines $\mu$ and $\pi$ momenta. The BH also measures the particle-separated beam fluxes.

Requirements:

The most stringent time resolution requirement is 100 ps needed for the reaction identification at the highest beam momentum; 80 ps has been achieved. High efficiency of $99 \%$ is needed to efficiently collect data and reject backgrounds; $99.8 \%$ has been achieved. A rate capability of $\approx 3.3 \mathrm{MHz}$ is needed to obtain the adequate statistical precision; the use of multiple paddles allows rates up to $10 \mathrm{MHz}$.

Design:

Figure 3 shows a $\mathrm{BH}$ plane under construction. We use BC-404 scintillator paddles that are $10 \mathrm{~cm}$ long and $\times 2 \mathrm{~mm}$ thick. Six central $4 \mathrm{~mm}$ wide paddles are flanked on each side by 5 outer 8 -mm wide paddles. The paddles are read out at each end by Hamamatsu S13360-3075PE SiPMs. A $6 \mu \mathrm{m}$ gap between the paddles suppresses cross-talk. To minimize effects on the beam and to achieve the needed per-

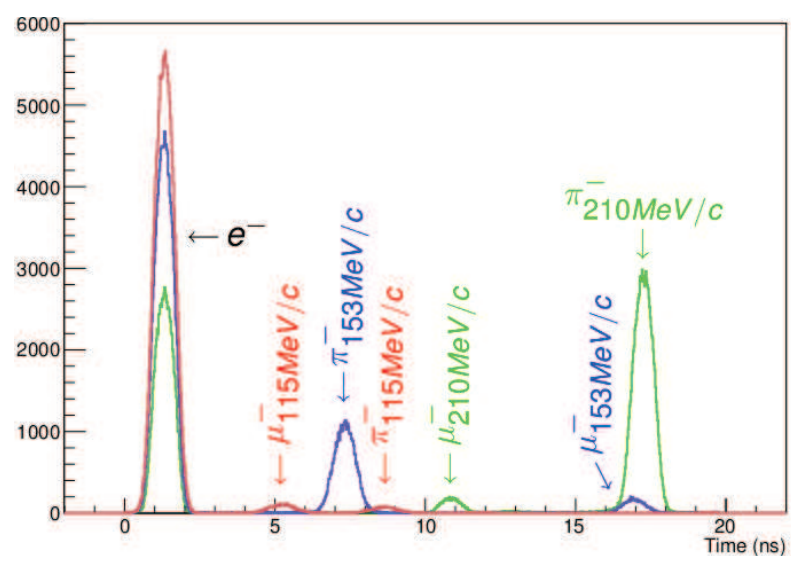

Fig. 1. Measured RF time spectrum for negative charge particles at 115,153 , and $210 \mathrm{MeV} / \mathrm{c}$. The spectrum wraps around every $\approx 20 \mathrm{~ns}$

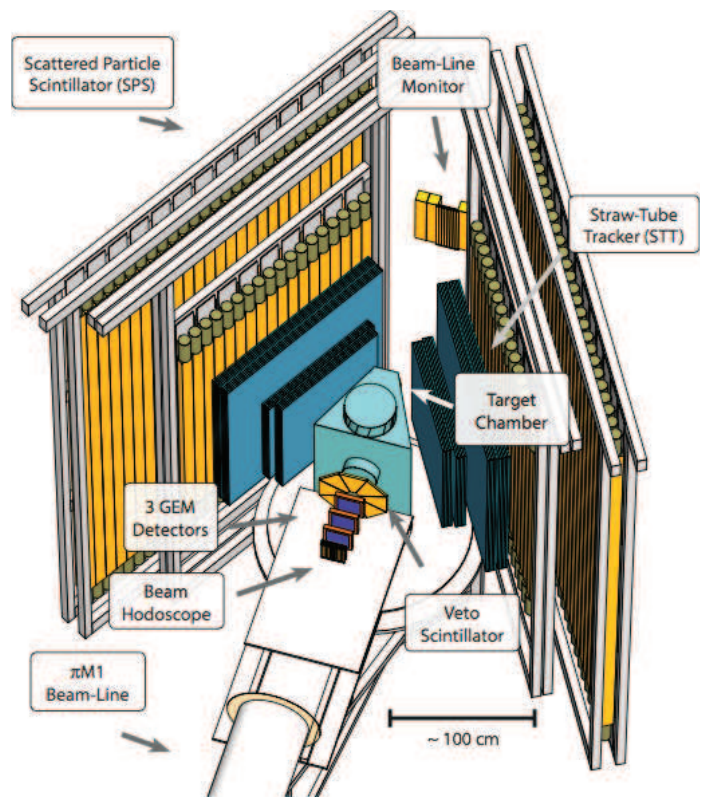

Fig. 2. MUSE detector setup implementation in GEANT4 simulation

formance, we use 2-4 planes of $\mathrm{BH}$ depending on the beam momentum.

The $\mathrm{BH}$ analog signal is amplified to produce a fast signal with a $1.2(3.0)$ ns rise (fall) time and typically a few hundred $\mathrm{mV}$ peak. A Mesytec CFD sends an analog copy of the signal to a Mesytec QDC input, and the discriminated signal to a TRB3 TDC and the trigger, and an OR of all input signals to the Mesytec QDC gate. 


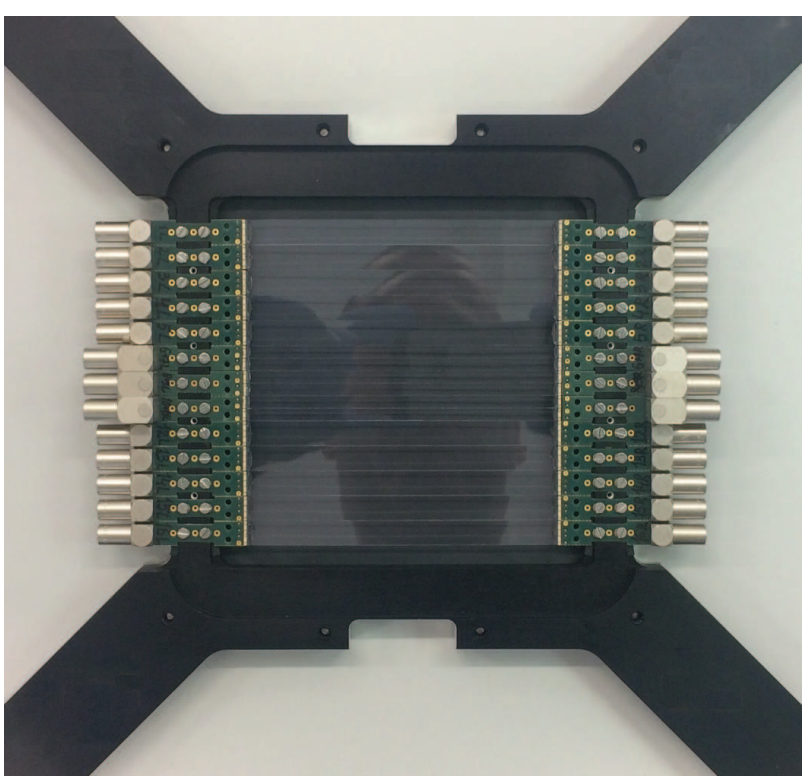

Fig. 3. BH plane with SiPM readout under construction

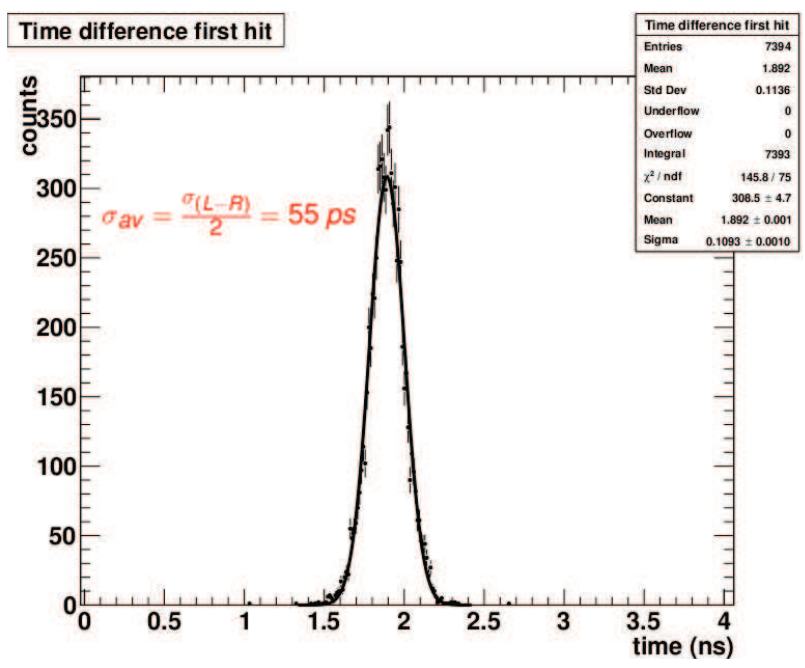

Fig. 4. The best obtained time resolution of a $\mathrm{BH}$ paddle

\section{Status:}

Five BH planes are built with all paddles meeting requirements. The best time resolution achieved is shown in Fig. 4.

\subsection{GEM trackers}

Purpose:

The GEMs measure beam trajectories so that precise scattering angles and reaction vertices can be determined. GEM chambers were chosen, as they are low-mass detectors, $\approx 0.5 \%$ of radiation length, which keeps the multiple scattering at a minimum, provide several $\mathrm{MHz}$ rate capability with $<100 \mu \mathrm{m}$ position resolution and high efficiency, $>98 \%$.

Requirements:

In addition to the performance characteristics given above, we require a read-out time $<100 \mu$ s for the efficiency in obtaining data.

Detector design:

We use three $10 \times 10 \mathrm{~cm}^{2}$ GEMs, which were previously used in OLYMPUS [10]. The GEMs are read out using FPGA-controlled frontend electronics based on the APV-25 chip developed for CMS and digitized with the Multi-Purpose Digitizer (MPD) v4. There are readout strips in two directions, each with $400 \mu \mathrm{m}$ pitch, much smaller than the amplified charge, which is distributed in a few mm wide cluster. Centroid weighting provides a resolution smaller than the pitch.

The GEM efficiency remains high at rate densities up to $2.5 \mathrm{MHz} / \mathrm{cm}^{2}$. The expected rate density for MUSE is $\approx 3.3 \mathrm{MHz} / 5 \mathrm{~cm}^{2}=0.66 \mathrm{MHz} / \mathrm{cm}^{2}$.

Current status:

The GEM system has been re-established for MUSE. We have implemented the new INFN/JLab DAQ readout software and VME controllers, which improve the read out speed along with low-noise operation and high efficiency reproduction. All requirements are now satisfed. The $\pi \mathrm{M} 1$ beam spot obtained by the GEMs is shown in Fig. 5 .

\subsection{Beam Veto}

Purpose and requirements:

The Beam Veto detector is used to reduce the trigger rate, by vetoing some of the events that arise from beam particle decays in flight or the scattering upstream of a scattering chamber. The veto detector requires a high efficiency, $>99 \%$, and a $1-n s$ time resolution - 200 ps has been achieved.

Design:

The Beam Veto detector uses the same technology as the SPS (described in Section 3.7), with a modified geometry and only single-ended readout. Figure 6 shows the detector. The detector geometry is nearly annular, surrounding the beam. Four trapezoidal BC404 scintillators are each read out with two Hamamatsu R13435 PMTs. The inner aperture roughly matches the target vacuum chamber entrance win- 


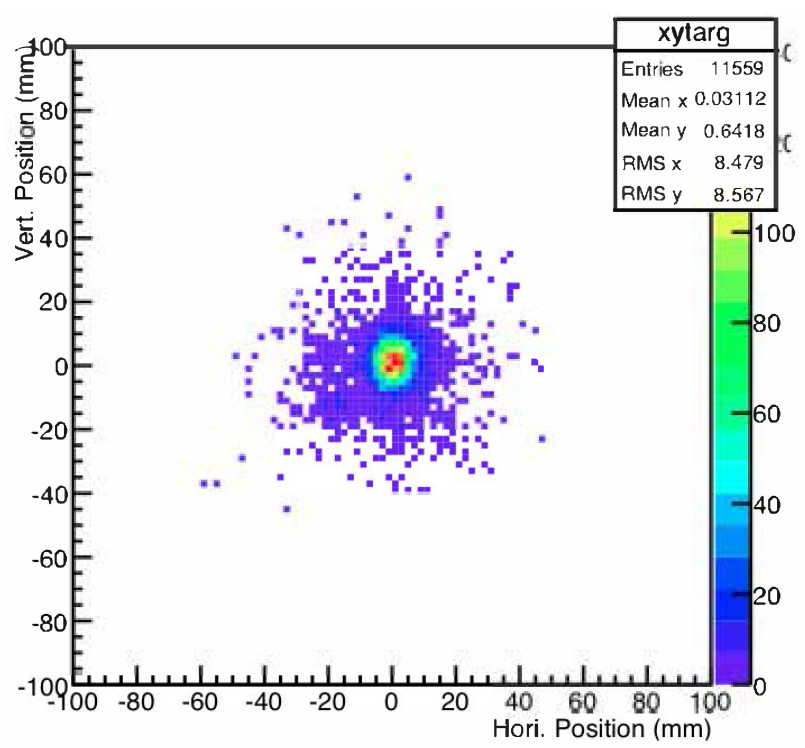

Fig. 5. The $\pi \mathrm{M} 1$ beam spot at the most upstream GEM detector

dow. The outer extent of the detector, about $16 \mathrm{~cm}$ radius, was determined from simulations.

Status:

The Beam Veto detector was built, installed, and commissioned. All performance requirements have been achieved.

\subsection{Liquid hydrogen target}

Purpose and requirements:

A Liquid Hydrogen $\left(\mathrm{LH}_{2}\right)$ target is needed for the $e p$ and $\mu p$ scatterings. In practice this requires a target ladder that includes at least a full cell, an empty cell for background subtractions, a solid target for alignment, and an empty position. The $\mathrm{LH}_{2}$ density must be stable, $<0.1 \%$, to precisely compare crosssections measured at different times. The geometry of the target must be uniform at the sub-mm level for precise background subtractions.

Design:

Detailed final construction designs and the actual construction were performed by CREARE Inc. working with the collaboration. The target ladder is housed in a vacuum chamber with a $7-\mathrm{cm}$ diameter entrance $(7.8 \mathrm{~cm}$ wide by $35.6 \mathrm{~cm}$ high exit) window, made of $125-\mu \mathrm{m}$ thick kapton. Scattered particles with $\theta=20^{\circ}-100^{\circ}$ go through side windows $33.7 \mathrm{~cm}$ wide and $35.6 \mathrm{~cm}$ high, made of Mylar lami-

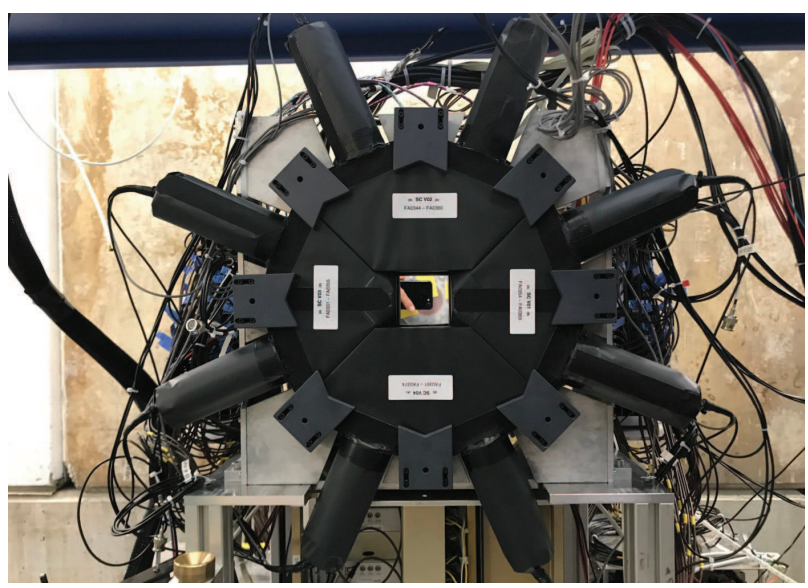

Fig. 6. Beam-Veto detector. The beam is pointing to us

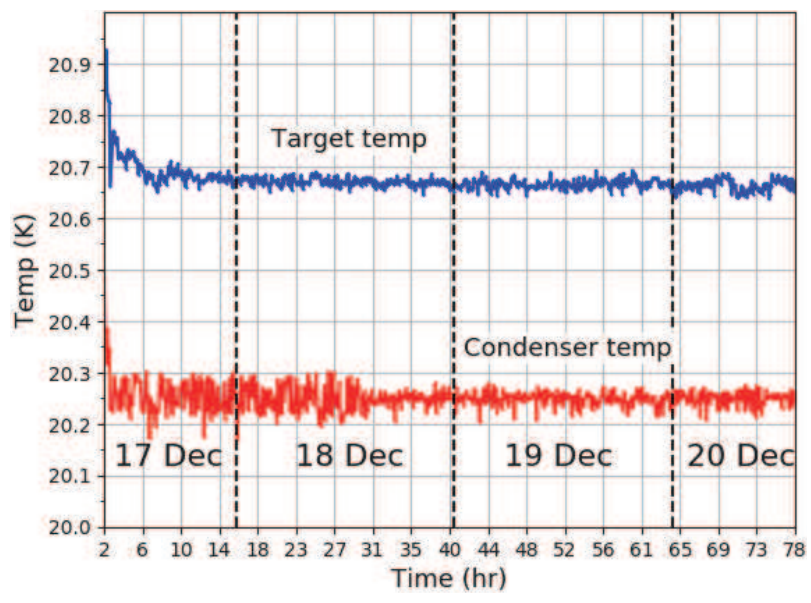

Fig. 7. Results of the first hydrogen cooldown, covering from 2 till 78 hours. Temperature stability assures the target density stability below the experimental requirements

nated on aramid sailcloth fabric, with an areal density

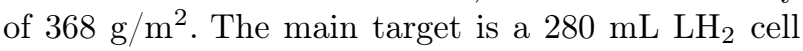
made of upper and lower copper end caps connected by a side wall of 4 wraps of $25 \mu \mathrm{m}$ thick kapton. A cryocooler cools a condenser assembly so that the resulting $\mathrm{LH}_{2}$ drips into the target cell. A lifting mechanism switches between ladder positions, lifting as well the cryocooler cold head and the condenser assembly. Current status:

Filling the cryotarget with LH2 at $20.67 \mathrm{~K}$ takes about $2.5 \mathrm{~h}$ from the start of the cooldown. The target was operated steadily for over three days at a pressure of 1.1 bar with temperature constant at the $0.01 \mathrm{~K}$ level - see Fig. 7 - consistent with the mea- 


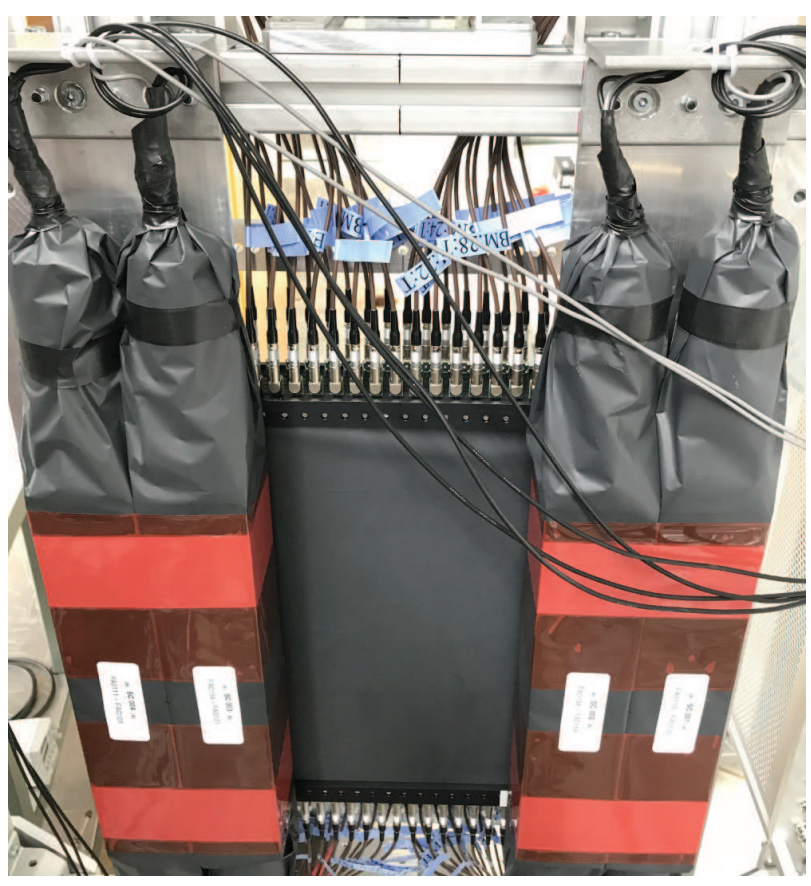

Fig. 8. Beam Monitor: The LEMO readout connectors of two offset planes are seen, continued with bigger BC-404 scintillators. The beam hits into the picture

surement uncertainty. This gives a target density of $0.070 \mathrm{~g} / \mathrm{cm}^{3}$ with stability $<0.02 \%$, better than experimental requirements.

\subsection{Beam monitor}

\section{Purpose:}

The BM provides a high-precision time measurement that determines the beam flux downstream of the target. TOF from the $\mathrm{BH}$ to $\mathrm{BM}$ determines a particle type and the background information for the RF-time only determination. TOF also determines $\mu$ and $\pi$ momenta to $<0.2 \%$. The BM also detects forward Moller electrons, so it can suppress this background in the scattering data.

Requirements:

The BM comprises a central scintillator hodoscope similar to the BH of Subsection 3.1 and an outer hodoscope similar to the SPS of Subsection 3.7. The underlying technology and requirements are the same in both cases.

Design:

Figure 8 shows the BM. The central hodoscope of the BM comprises two offset planes of 16 paddles. We use $30 \mathrm{~cm}$ long $\times 12 \mathrm{~mm}$ wide $\times 3 \mathrm{~mm}$ thick BC-404 paddles, each read out at each end by 3 Hamamatsu S13360-3075PE SiPMs in series. The same readout electronics as for the $\mathrm{BH}$ is used. The outer hodoscope consists of four $30 \mathrm{~cm}$ long $\times 6 \mathrm{~cm}$ wide $\times 6 \mathrm{~cm}$ thick BC-404 scintillator paddles that are identical in technology to the SPS scintillators.

Status:

The BM was fully assembled, installed, and commissioned. Typical time resolutions of $\sigma_{T}<100 \mathrm{ps}$ (Best: $\sigma_{T}=59 \mathrm{ps}$ ) were achieved, with $\geq 99.9 \%$ efficiency, exceeding performance requirements.

\subsection{Straw-tube tracker}

Purpose and requirements:

The STT tracks scattered particles. High resolution, $<150 \mu \mathrm{m}$, and efficiency, $>99.8 \%$, are required for precise cross-sections.

Design:

The STT follows the PANDA straw chamber design [11] adapted to the MUSE geometry. We use the same straws, wires, end pieces, and feed-throughs as PANDA. Thin-walled, over-pressured straws allow for a significantly less straw material, while providing the mechanical stability. The straw spacing is $1.01 \mathrm{~cm}$, and adjacent offset straw planes are $0.87 \mathrm{~cm}$ apart.

The symmetric beam left and right scattered particle detector systems include 2 chambers on each side of the beam, each with 5 vertical and 5 horizontal planes, to achieve a high tracking efficiency. The front chambers have 27560 -cm long vertical straws and 300 $55-\mathrm{cm}$ long horizontal straws, with an active area of $60 \times 55 \mathrm{~cm}^{2}$. The rear chambers have $40090-\mathrm{cm}$ long vertical straws and $45080-\mathrm{cm}$ long horizontal straws with an active area of $90 \times 80 \mathrm{~cm}^{2}$. The front (rear) chambers are $30(45) \mathrm{cm}$ from the target. There are 2850 straws in the system. The STT uses $90 \% \mathrm{Ar}$ $+10 \% \mathrm{CO}_{2}$ at a pressure of 2 bar. Straws operate at $1700 \mathrm{~V}$. Frontend PASTTREC cards read out the straws, and are in turn read out by TRB3 TDCs.

Status:

All 4 chambers have been assembled at PSI and undergone initial performance tests. Figure 9 shows the STT being craned into the MUSE detector setup. In the initial tests, the straws operated reliably with approximately $90 \%$ efficiency, which yields $\approx 99 \%$ tracking efficiency for 5 planes. A preliminary analysis of 


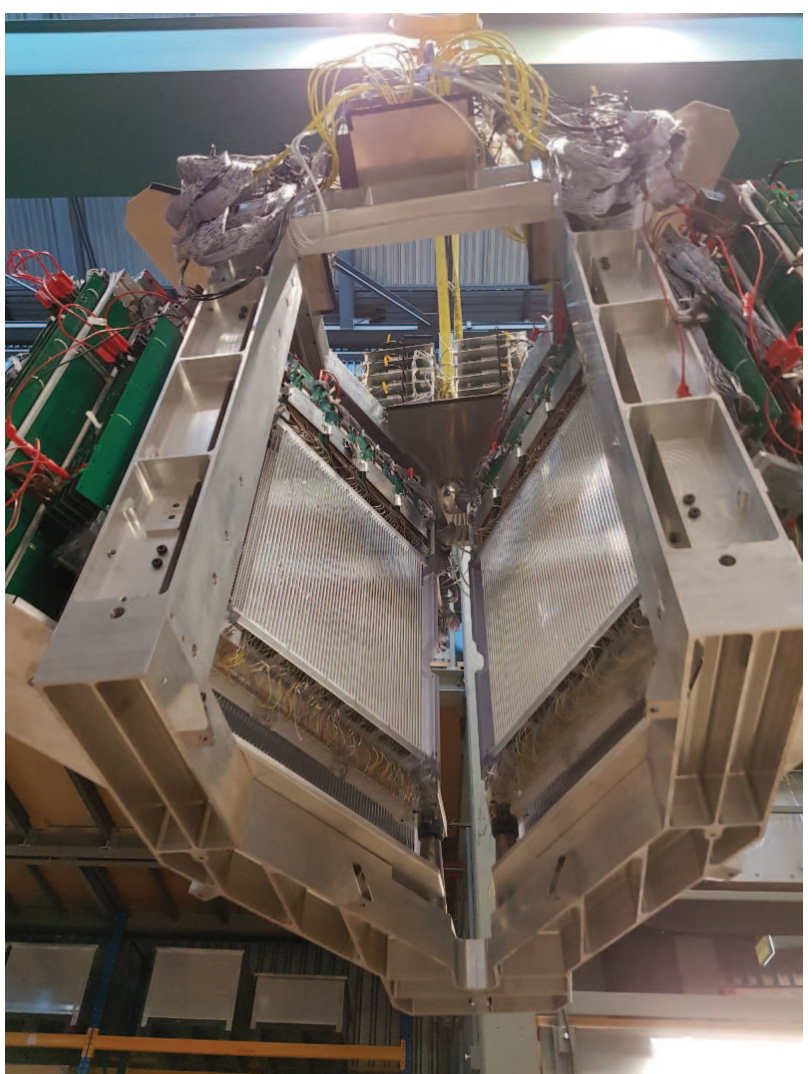

Fig. 9. Fully assembled STT detector being craned into the MUSE detector setup

the STT data yields a tracking resolution of approximately $115 \mu \mathrm{m}$, exceeding requirements.

\subsection{Scattered particle scintillators}

Purpose and requirements:

The SPS is a high-efficiency high-precision scintillator hodoscope that detects and times particles for the triggering and reaction identification. A time resolution of $\approx 100 \mathrm{ps}$ is needed for the reaction identification. A uniform efficiency of $>99 \%$ is needed so that the shape of the angular distribution is not altered. Design:

The SPS design follows the JLab CLAS12 FTOF12 design by University of South Carolina. Symmetric left and right, front and rear hodoscope paddles are made of an Eljen Technology EJ-204 plastic scintillator, which has a high light output and a fast rise time. Hamamatsu R13435 PMTs are glued to each end of the scintillator. The front wall is roughly square and ISSN 2071-0194. Ukr. J. Phys. 2019. Vol. 64, No. 7

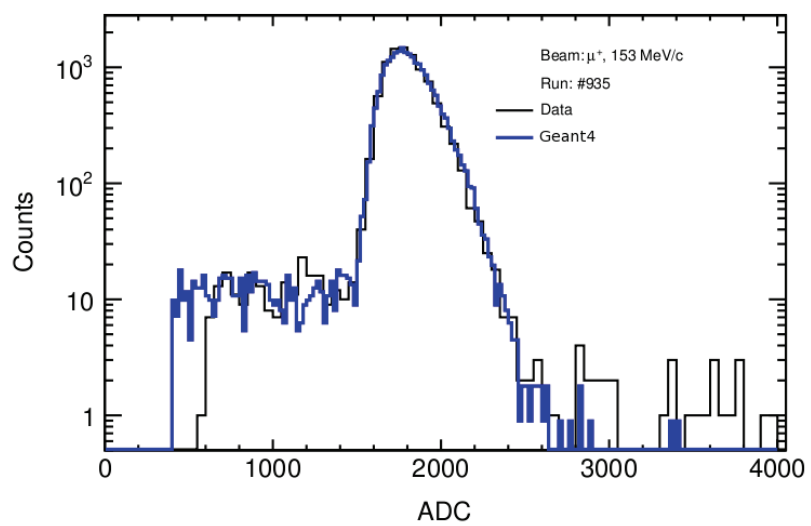

Fig. 10. SPS ADC spectra: Particles stopping or going through the bar are in peak; particles going out the side of the bar are in the low-energy tail

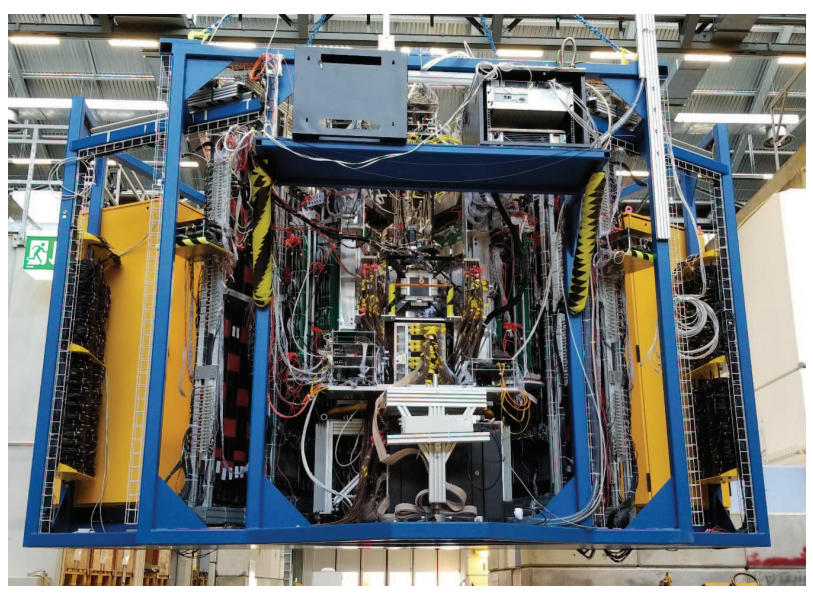

Fig. 11. MUSE Detector setup is being craned into the experimental area

covers $\theta \approx 20^{\circ}-100^{\circ}$. The oversized back wall accounts for the multiple scattering in the front wall.

Status:

All 92 paddles are tested and installed. Average time resolutions of $\sigma_{\mathrm{avg}}=52 \mathrm{ps} \pm 4 \mathrm{ps}$ for the 220 -cm long rear bars and 46 ps \pm 4 ps for the 110 $\mathrm{cm}$ long front bars were obtained. Figure 10 shows that energy deposition in the scintillators modeled with Geant4 simulations agrees nicely with measurements.

The two-plane coincidence efficiency is well above $99.5 \%$, except for $e^{+}(\geq 99 \%)$ due to the annihilation. We expect the cross-section systematic uncertainty from the SPS efficiency to be $<0.1 \%$. 


\subsection{Trigger, $D A Q$, and tracking}

MUSE uses TRB3 FPGAs for the triggering. The primary scattered particle trigger logic is:

$\left(e^{ \pm} \mathrm{OR} \mu^{ \pm}\right) \mathrm{AND}\left(\right.$ no $\left.\pi^{ \pm}\right)$AND (scatter) AND (no Veto).

The MUSE DAQ uses a mix of VME modules for the charge determination and TRB3 TDCs for the timing. There are about $3000 \mathrm{TDC}$ and $500 \mathrm{Q} / \mathrm{ADC}$ channels. Both the trigger and DAQ along with controls and basic analysis software are fully operational. The advanced analysis software development continues.

\section{Conclusions}

The data taken compared to simulations prove that MUSE is well suited to investigate the $\left(R_{e}-R_{\mu}=\right.$ $=0.034 \pm 0.006 \mathrm{fm}) 5.6 \sigma$ Proton Radius Puzzle. By comparing the $p+e^{ \pm}$and $p+\mu^{ \pm}$scattering crosssections, we will determine the absolute radius at the $\approx 0.005 \mathrm{fm}$ level. All detectors are constructed and mounted on the MUSE platform. Production data runs are planned in 2019-2021.

We acknowledge the US National Science Foundation for supporting the MUSE experiment, with the additional support from the US Department of Energy, the Binational Science Foundation, and the Paul Scherrer Institute. Special thanks to Prof. Dr. Ronald Gilman for creating such a nice working atmosphere for me. Thanks to PSI for the great hospitality and the organizers of the "New Trends in High Energy Physics" for giving me opportunity to present this work.

1. R. Pohl, A. Antognini, F. Nez, F.D. Amaro, F. Biraben, et al. The size of the proton. Nature 466, 213 (2010).

2. A. Antognini et al. Proton structure from the measurement of $2 \mathrm{~S}-2 \mathrm{P}$ transition frequencies of muonic hydrogen. Science 339, 417 (2013).

3. J. Bernauer et al. (A1 Collaboration). High-precision determination of the electric and magnetic form factors of the proton. Phys. Rev. Lett. 105, 242001 (2010).
4. X. Zhan, K. Allada, D. Armstrong, J. Arrington, W. Bertozzi et al. High-precision measurement of the proton elastic form factor ratio $\mu \mathrm{pGE} / \mathrm{GM}$ at low Q2. Phys. Lett. B 705, 59 (2011).

5. R. Pohl, R. Gilman, G. Miller, K. Pachucki. Muonic hydrogen and the proton radius puzzle. Annu. Rev. Nucl. Part. Sci. 63, 175 (2013).

6. R. Pohl, G.A. Miller, R. Gilman et al. Workshop on the proton radius puzzle at the Trento European Center for Theory in Nuclear Physics and Related Areas (2012) https://ectstar.fbk.eu/node/49.

7. C. Carlson, R. Hill, S. Karshenboim, M. Vanderhaeghen et al. Workshop on the proton radius puzzle at the Mainz Institute for Theoretical Physics (2014).

8. R. Pohl, G. A. Miller, R. Gilman et al. Workshop on the proton radius puzzle at the Trento European Center for Theory in Nuclear Physics and Related Areas (2016) https://ectstar.fbk.eu/node/1659.

9. S. Agostinelli et al. GEANT4: A Simulation toolkit. Nucl. Instrum. Meth. A 506, 250 (2003).

10. R. Milner et al. The OLYMPUS experiment. Nuclear Inst. and Methods in Physics Research A 741, 1 (2014).

11. W. Erni et al. Technical design report for the $\bar{P}$ ANDA (AntiProton Annihilations at Darmstadt) straw tube tracker. Europ. Phys. J. A 49, 1 (2013).

Received 08.07.19

\section{Т. Ростомян}

\section{СТАТУС ЕКСПЕРИМЕНТУ MUSE}

Р е $з$ ю м е

Різниця в $5.6 \sigma$ у радіусах протона, виміряних 3 атомами $\mu p$ і з атомами ер та в процесі розсіяння залишається нерозв'язаною головоломкою. В проекті MUSE буде вимірюватися пружне розсіяння $\mu p$ і ер в тому самому експерименті одночасно. Експеримент визначає перерізи, двофотонні ефекти, форм-фактори та радіуси, і дозволяє порівнювати результати, отримані для $\mu p$ і ер процесів зі зменшеною систематичною похибкою. Ці дані повинні забезпечити найкращий на сьогоднішній день тест універсальності лептона в процесі розсіяння, на порядок поліпшений у порівнянні 3 попередніми тестами, дати можливість визначити радіус 3 інтервалом надійності $7 \sigma$ і забезпечити покращені двофотонні вимірювання. 